\title{
EM analysis of meiotic chromosome pairing in a pentaploid Achillea hybrid
}

\author{
J. Loidl, \\ F. Ehrendorfer and \\ D. Schweizer
}

Institute of Botany, University of Vienna, Rennweg 14, A-1030 Wien, Austria

Presynaptic alignment occurs at zygotene and early pachytene in a pentaploid hybrid between Achillea collina (4x) and A. millefolium (6x). Mostly, the five corresponding chromosomes of each set are assorted into a group of three (two synapsed plus one aligned) and a group of two (synapsed); groups of five axes by alignment and/or pairing are extremely rare. This reflects different degrees of homology between the five genomes and allows inferences about the possible phylogeny and genome formulas of the tetraploid (AASS) and hexaploid (AASSTT) involved. The alignment as subgroups of three and two chromosomes in their $5 \boldsymbol{x}$ hybrid also indicates that synaptic alignment responds to slightly different degrees of homology and is decisive regarding which chromosomes will finally pair effectively and cross over. At early zygotene, alignment is weakly expressed, arguing against premeiotic associations as a precondition for homologous alignment and subsequent synaptonemal complex formation. At late pachytene, synapsis is maximized by autosynapsis of chromosomes which do not take part in the preceding homologous two-by-two synapsis.

\section{INTRODUCTION}

It is common belief that the formation of the synaptonemal complex (SC) is preceded by an alignment of homologous chromosomes. This alignment is not obvious in common diploid meiosis because from zygotene on it is concealed by the formation of the SC. Only in cases where there are supernumerary homologous chromosomes which are excluded from synapsis, can their alignment be observed far into pachytene (e.g., Loidl and Jones, 1986). Rasmussen (1977) noted that triploidy allows a clear distinction between the primary recognition of homologues and their intimate pairing through the SC.

As in triploids, alignment to a higher or lesser degree should be visible in all instances where more than two homologues are present, and where, as a consequence, homology does not result in synapsis between all of them. In the present paper this situation is studied in a pentaploid Achillea where various degrees of affinity between the five chromosome sets seem to exist. Hence it is of interest to see whether differences in the frequency and extent of alignment result from the different similarities of the genomes involved.

There is a dispute over the problem of when "primary recognition", as indicated by alignment, takes place (see e.g., Gillies, 1984; Wettstein et al., 1984 for discussion). The first appearance of presynaptic alignment could be taken as indicative whether the first homologous encounter takes place during meiotic prophase, preceding SC initiation only shortly, or if it dates back to premeiotic interphase or even before.

\section{MATERIALS AND METHODS}

A single plant of yarrow, transplanted from the NE Limestone Alps of Lower Austria (Schneeberg, Sparbacher Hütte, mountain pasture at $c a .1250 \mathrm{~m}$ ) to the Botanical Garden of the University of Vienna (HBV), was used. This individual was hyperpentaploid $(5 x+1,2 n=46)$ and grew among a population of tetraploid Achillea collina ( $4 x$, $2 n=36$ ); nearby also hexaploid A. millefolium (6x, $2 n=54$ ) occurs. In other contact zones between these two microspecies $5 x$ F1-hybrids and aneuploid backcross individuals are not rare. An experimental analysis of such hybrids has been presented by Schneider (1958). The $5 x+1$-plant studied here is morphologically intermediate between $A$. collina and $A$. millefolium and thus must be regarded as a hybrid (with the additional chromosome possibly from non-disjunction, either during gamete forma- 


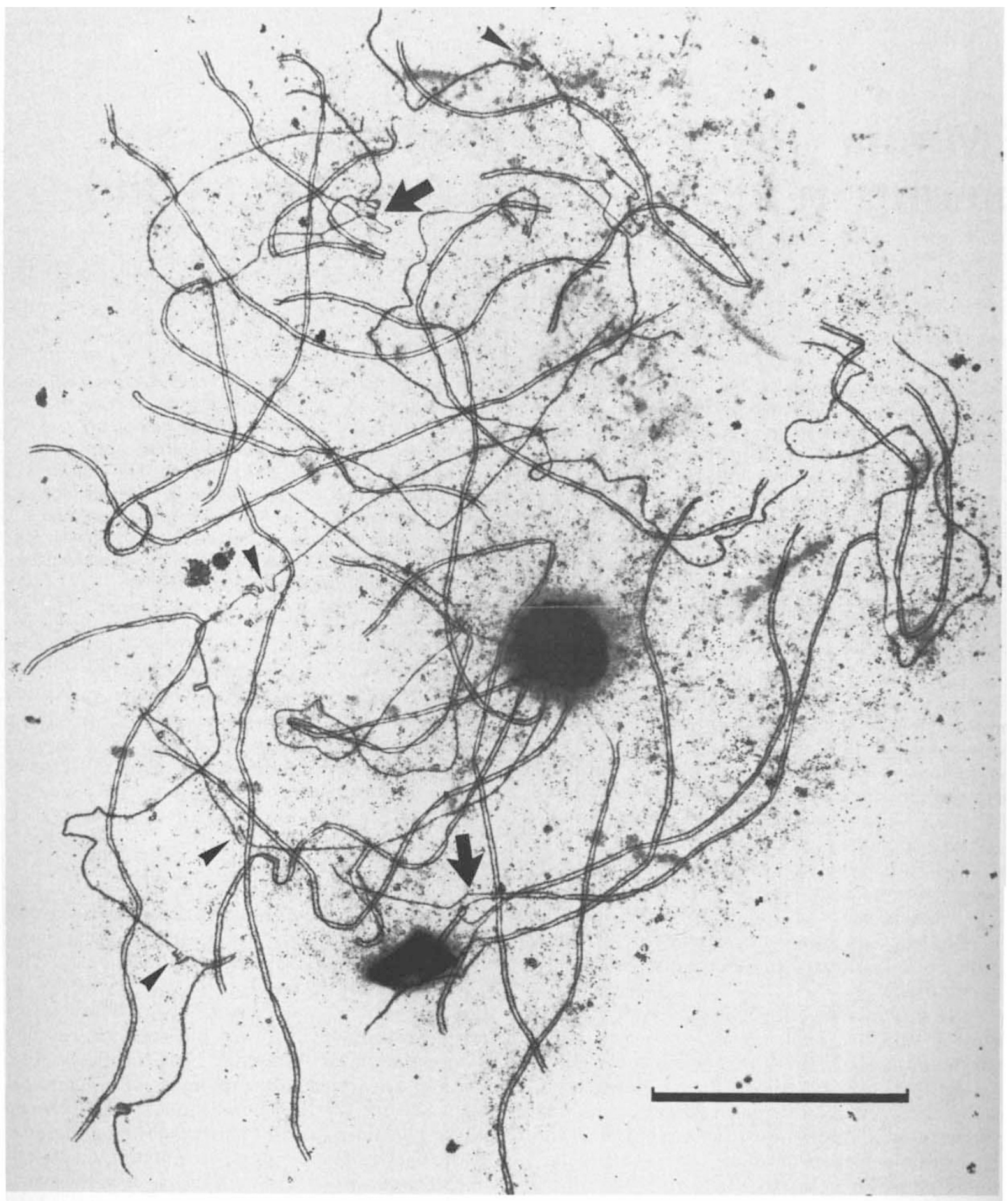

Figure 1 Electron micrograph of a pachytene nucleus of Achillea $(5 x+1)$ corresponding with nucleus $I$ in fig. 4 . Several triplets consisting of two synapsed plus one aligned chromosomes can be recognized. Arrows point to pairing partner switches between three and six chromosomes. Unsynapsed axial elements show several discontinuities (arrowheads). About one third of a bivalent at the lower edge is cut away. Bar represents $10 \mu \mathrm{m}$. 
tion or during embryo development). Its phytochemical profile (Greger, unpublished) does not contradict such an origin.

Whole mount preparations of synaptonemal complexes (SCs) were made from pollen mother cells (PMCs) by a spreading method according to Albini and Jones (1984). The basic procedure, including enzymatic digestion of pollen mother cell walls, spreading of the meiocytes with a detergent and fixation of the preparations with 4 per cent paraformaldehyde, was employed practically unaltered. Because of the smallness of the anthers about 20 buds of appropriate size from several partial inflorescences were collected for the production of one preparation. For spreading a solution of 1 per cent "Lipsol" (twice the concentration given in the original schedule) was used.

Silver staining was done according to Loidl and Jones (1986) and for the transfer of slides into electron microscopic preparations the schedule given by Loidl (1989) was followed.

For evaluation individual configurations from enlarged EM micrographs were traced on transparent paper to facilitate identification, and measured using a digitizer.

\section{RESULTS}

From the 63 PMC nuclei screened in the EM, eight complete pachytene nuclei (as shown in figs 1 and 2) were measured. The sum of axial/lateral elements per nucleus amounts to $1406,1447,1479$, $1489,1510,1556,1580$ and $1581 \mu \mathrm{m}$, respectively. These values are remarkably homogeneous for spread SCs where not only stage-specific condensation but also the preparation method may contribute to varying SC lengths (Loidl, 1986, 1988). Lateral/axial elements are well preserved, nucleoli are usually present as very dense spots. Central elements were seen only in some instances and centromeres are sometimes visible at the beginning of diplotene when SCs start to disintegrate.

There are many instances where the axes of univalents show apparent overlapping discontinuities attached to what appear to be short pieces of central element (fig. 1). A closer look at some of these structures reveals, however, that they result from S-shaped double-foldbacks where the axes are broken at the points of inflection (fig. 3(a,b)).

The majority of nuclei (51 out of 63 ) were at (near-) complete pachytene. The earliest PMCs studied were at early zygotene and showed some presynaptic alignment of a few regions only (fig.
$3 c)$. During the course of zygotene, not only the synapsed but also the aligned portions of axes increase. Unlike in autopentaploid Allium oleraceum (Loidl, 1988), alignment does not take place between all five chromosomes of an allied group. Instead, there is a certain preference for the formation of triplets (three axes grouped together by alignment and/or pairing switches) plus bivalents (fig. 4), which probably reflects different degrees of homology between the chromosomes of an allied group of five. Only 8 quintets (i.e., five axes bundled) as compared to 50 triplets were found in a sample of easily analysable configurations collected from several nuclei. However, even the chromosomes within triplets do not seem to be equally homologous. Compared to autotriploid Allium sphaerocephalon (Loidl and Jones, 1986), alignment within triplets is much less intimate and, probably as a consequence, the frequency of pairing switches is much lower. In Achillea, among 50 triplets there were 14 trivalents (13 with one, one with two pairing switches); in the remaining triplets the third axis was merely aligned. Thus there are fewer pairing switches than expected from a competitive pairing situation between three homologous chromosomes. Provided that there are at least two initiation sites per configuration (which according to zygotene observations may be assumed for Achillea) there should be twice as many trivalents as groups of bivalents plus univalents (e.g., Sybenga, 1975, p. 121). It is suggested, therefore, that triplets consist of a pair of homologous axes and one merely homoeologous axis.

Of the eight quintets two were pentavalents with two pairing switches each. Moreover, one quadrivalent was observed (with one pairing switch), and one hexavalent with two pairing switches. The latter indicates the presence of six allied chromosomes. It is conjectured that a chromosome has doubled due to non-disjunction in the meiosis of one parent or during embryo development, leading to a hyperpentaploid $(5 x+$ 1) karyotype which was confirmed by chromosome counts in root-tip mitoses. Correspondingly, in all eight completely analysed cells the sum of chromosome axes was 46 (see fig. 4).

The allied group which contains the excess chromosome seems to preferentially form three bivalents at pachytene, whereas, due to the uneven number of chromosomes, one axis is always single in the other allied groups. In the cells depicted in fig. 4, all but one of the single axes are either aligned (indicated by horizontal dotted lines) with their synapsed homoeologues or involved in 
pairing switches. The free single axes (drawn to the right) show various degrees of hairpin formation. The structure, designated e, in fig. 4 does not appear to be a hairpin at first sight because it is open at both ends and hence resembles a zygotene bivalent. However, it is too short for a bivalent, and elsewhere in this material it could be seen that axial elements tend to break where they are bent back sharply on themselves (see above). Therefore it is justified to conclude that this configuration results from the autosynapsis of a univalent.
Single axes are always longer than their paired homoeologous partners with which they are aligned. This situation is paralleled by that in triploid Allium sphaerocephalon (Loidl and Jones, 1986). In Achillea, 47 groups of closely aligned triplets at pachytene were measured and it turned out that the length ratio of synapsed to unsynapsed axes was $1: 1 \cdot 44 \pm 0 \cdot 14$ ).

Axial elements that were not involved in the preceding homologous pairing showed a tendency to accomplish non-homologous synapsis by intercalary and terminal foldbacks. This is more evident

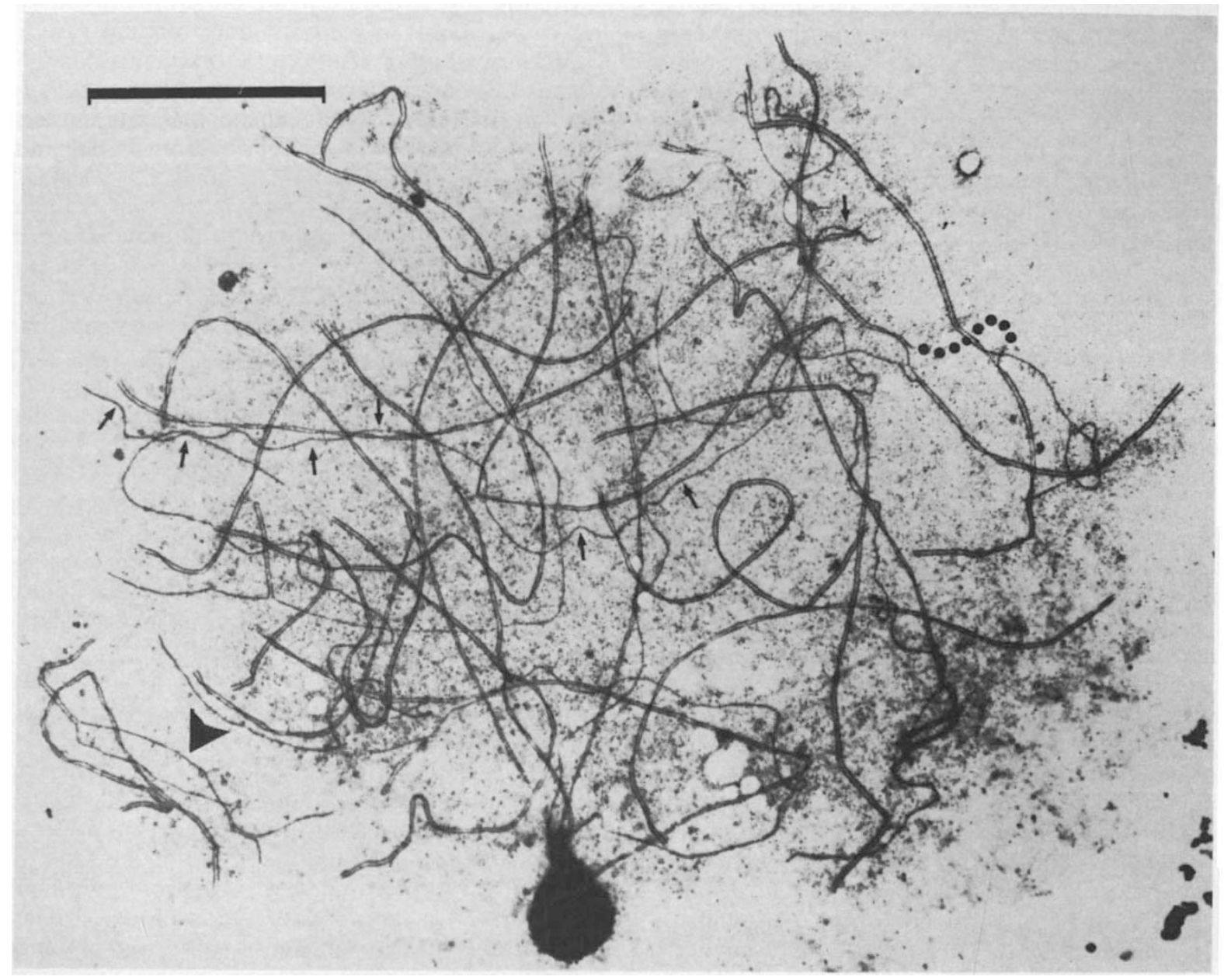

Figure 2 Pachytene nucleus corresponding with nucleus II in fig. 4. Dotted line indicates a break of a lateral element at a pairing switch in a pentavalent. Arrows denote a univalent which is aligned with two different bivalents. The configuration marked by an arrowhead is likely to represent a univalent which is folded back on itself. Bar represents $10 \mu \mathrm{m}$. 


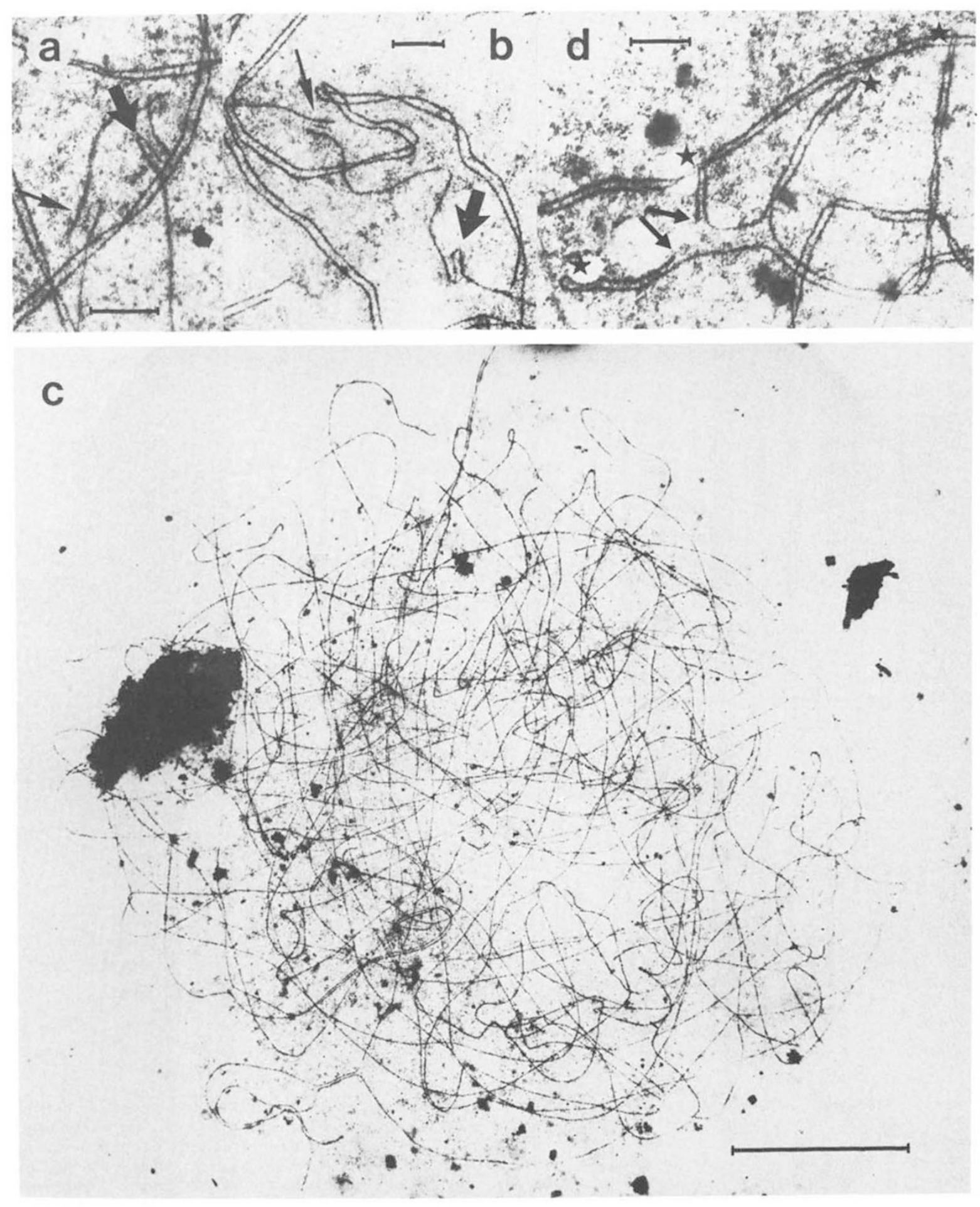

Figure 3 Electron micrographs of spread Achillea SCs. (a) and (b) show discontinuities within axial elements (thin arrows) and at the same time illustrate their possible origin as short S-shaped paired loops (thick arrows). (c) Early zygotene at which some presynaptic alignment can be recognized and (d) a univalent, presumably at late pachytene, which is completely autosynapsed via four hairpin loops (asterisks). Arrows mark the ends of the axis. Bars in (a), (b) and (d) $1 \mu \mathrm{m}$, in (c) $10 \mu \mathrm{m}$. 

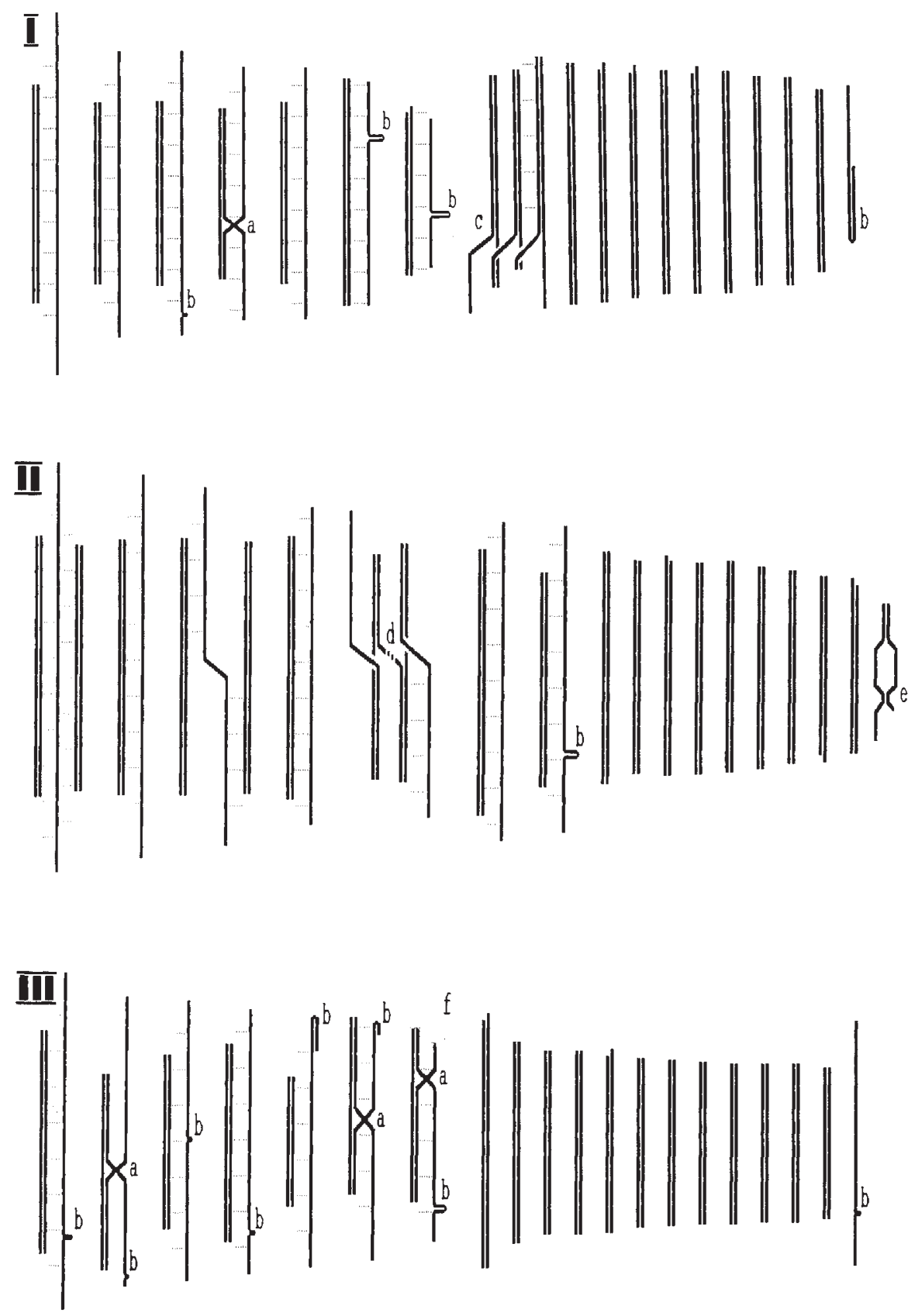

Figure 4 Schematical drawing of aspects of alignment and pairing in three pachytene nuclei. Lateral/axial elements are ordered according to size in groups of multiple configurations, bivalents and univalents. Relative lengths within and between the nuclei I, II and III are reproduced correctly. Transverse dotted lines indicate alignment. In nucleus I there are seven triplets (including one trivalent), one hexavalent, nine bivalents and one free univalent. In nucleus II there are three quintets (including one pentavalent), four triplets, nine bivalents and one free univalent. In nucleus III there are seven triplets/trivalents, 12 bivalents and one free univalent. In all three nuclei the sum of axial/lateral elements is 46 , consistent with a hyperpentaploid $(5 x+1)$ karyotype. (a) Pairing switches in trivalents. (b) Terminal and intercalary foldbacks (hairpins). (c) Hexavalent. (d) Pentavalent; one lateral element is disrupted at a switch. (e) Extensive foldback pairing in a univalent; the axial element is broken at the top where it bends back on itself. (f) Nucleous which conceals the course of axes. Note that unsynapsed (portions of) axes are considerably longer than their homologous/homoeologous counterparts. Note also that alignment is not necessarily concomitant with multivalent formation (it may have been partially abolished at that stage already or been disrupted by the spreading). 
in axes that are not aligned with their homoeologues (fig. 4).

Three out of the 63 nuclei screened showed almost complete (over 90 per cent estimated) synapsis of homologous as well as nonhomologous kind. These are interpreted as late pachytene and their low frequency may indicate that the duration of that stage is short. It is possible, on the other hand, that many nuclei enter diplotene without having achieved complete nonhomologous synapsis. Fig. 3(d) shows a single axis in such a nucleus, which is completely autosynapsed by forming several hairpin-loops. A similar phenomenon was described in autotriploid Allium sphaerocephalon by Loidl and Jones (1986) where virtually complete non-homologous synapsis of axes, excluded from homologous synapsis by pairing competition, takes place.

\section{DISCUSSION}

\section{The time of primary recognition}

Various concepts of somatic or premeiotic association of homologues as a means of ensuring preferential homologous pairing have been proposed (e.g., Brown and Stack, 1968; Grell, 1969; Maguire, 1977, 1988; Ashley and Pocock, 1981; Dickinson, 1987). The existence of premeiotic associations in the form of parallel alignment has been disputed (e.g., Palmer, 1971; Therman and Sarto, 1977; Del Fosse and Church, 1981; Bennett, 1984; Holm and Wang, 1988) on the grounds that it should be visible already before the leptotene/zygotene transition, but was not found. Also in the present study no alignment was found in most parts of early zygotene nuclei, although in Achillea alignment is a striking property of mid-zygotene and pachytene. Therefore, with a few notable exceptions like the dipterans (Wandall and Svendsen, 1985), the concept of premeiotic associations is unlikely to apply to several organisms investigated so far ultrastructurally, including Achillea. It cannot be ruled out, however, that weak connections exist between the chromosomes at stages previous to zygotene, which escape detection. Only the prolonged leptotene-like state of unsynapsed axes as found in polyploids could give them time to become more numerous and stronger and cause the alignment we see here.

\section{Non-homologous synapsis}

During pachytene, when the SC has taken over the contact between the homologues, alignment relaxes (Rasmussen, 1977; Loidl and Jones, 1986). The single axial elements at that stage have a continued readiness for pairing. This is also reflected by the tendency of univalents to remain attached to the nuclear envelope within the bouquet region longer than those of the bivalents, as observed by Rasmussen (1977). Concomitantly with their release from homologous/homoeologous alignment the univalents become engaged in non-homologous synapsis. In Achillea, terminal and intercalary foldbacks appeared during pachytene and in a few nuclei non-homologous autosynapsis was so extensive that almost no unsynapsed portions were left.

A tendency towards maximum SC-pairing, even if non-homologous, seems to be a common feature of haploids (e.g., Wang, 1988; see also Gillies, 1984), of the autosynapsis of B-chromosomes (Switoński et al., 1987), of XY bivalents (e.g., Tres, 1977; Joseph and Chandley, 1984), of X-univalents (Speed, 1986), of structural heterozygotes (e.g., Loidl, 1987; Sudman et al., 1989 and lit. cit. therein) and of many polyploids (Rasmussen 1977, Jenkins, 1985; Loidl and Jones, 1986). On the other hand, White et al. (1988) preferred internal homology due to duplicated DNA as an explanation for foldback loops in supernumerary segments of diploid Scilla autumnalis.

The fact that synapsis per se is indifferent to homology supports the concept of a recognition process which is concomitant with presynaptic alignment. Presynaptic alignment is sometimes imagined as a rather coarse mechanism that sorts out groups of homologues/homoeologues in order to prevent interlocking during the subsequent pairing for which homology requirements are more stringent. The present results, however, suggest that presynaptic alignment is a highly selective process which is capable of discriminating between different degrees of homology.

\section{Is the SC a signal for chromosome compaction?}

There are several ways to explain why the aligned but unpaired axes are always longer than their synapsed partners. One is that the spreading may cause a disproportionally stronger stretching of the unsynapsed axes. This is supported by the observation that in spreads of autotriploid Allium sphaerocephalon (Loidl and Jones, 1986) and in tetraploid Allium vineale (Loidl, 1986) the unsynapsed axes are longer, too, whereas in serial 
sections of triploid Bombyx oocytes the proportions are rather the reverse (Rasmussen, 1977). However, in Achillea and the other spread materials single axes showed no obvious signs of overstretching, like discontinuities or poorly staining regions. Another explanation for the length difference between single and paired homoeologous axes was suggested by Jenkins (1985) for triploid Lolium temulentum $\times$ perenne. The DNA amount of $L$. temulentum is 150 per cent of that of $L$. perenne. Part of this difference is reflected by the different lengths of the two synapsed $L$. perenne axes on the one hand, and the single $L$. temulentum axis on the other. (The fact that the length difference is lower than expected from the difference of genome sizes, Jenkins (1985) ascribes to differential compaction of the two chromosome sets prior to synapsis.) Similarly, in a triploid Scilla autumnalis, the greater length of the unsynapsed axes was attributed in part to the larger DNA amount and mitotic length of the cytotype that they represent (White et al., 1988).

For Achillea it can be ruled out that such considerable length differences between karyotypes exist that could be responsible for the greater length of single axes as observed. Firstly, length differences were not observed in metaphase chromosomes (Tohidast-Akrad and Ehrendorfer, unpublished); if they exist they must be quite small. Secondly, even when the three homoeologous axes are joined by a pairing switch, it is in each case the unsynapsed portion that is longer, irrespective of to which chromosome it belongs (see fig. 4).

Finally, length differences could occur because synapsed regions of the axes are more condensed than unsynapsed ones in the same nucleus. A progressive reduction in SC length from zygotene throughout pachytene has been reported for several organisms (see Gillies, 1984). Possibly, condensation is not steady and simultaneous but intermittent and takes place only in portions which have initiated synapsis already. This possibility invites the further speculation that homologous alignment (and the recognition processes involved therein) requires a less condensed state of chromosomes, and that condensation is resumed only after initiation of the SC and is concomitant with synapsis. According to this proposal the SC would serve as a signal for condensation.

The dependency of condensation on synapsis would also provide a ready explanation for the fact that synapsis is complete in most organisms even where chiasmata are localized (Jones, 1987) and for the tendency of axes to get involved in non-homologous synapsis if they fail to encounter a homologous partner before late pachytene (see above). It could be dictated by the need to undergo synapsis-directed condensation.

\section{Systematic implications of association behaviour}

The pairing situation in $5 x$ Achillea is not matched by those found in pentaploids so far. Allium oleraceum, investigated by Loidl (1988), is clearly autopentaploid, forming bundles of five aligned homologous chromosome axes at zygotene. The hybrids between Triticum aestivum $\times T$. kotschyi (Gillies, 1987) are essentially pentahaploids with only moderate homology between the five genomes and unclear pairing preferences. In Achillea, on the other hand, there is clear evidence for preferential alignment within sets of homoeologues which frequently form a group of three (two paired, one aligned) plus a group of two (mostly paired). This grouping invites the following interpretation. The pentaploid is a hybrid that has received three homoeologues from its hexaploid parent (Achillea millefolium s.str.) and two homoeologues from its tetraploid parent ( $A$. collina). (The particular case presented here is somewhat complicated by the $a$ posteriori doubling of a chromosome.) Pairing between the parental homoeologous groups seems to be largely suppressed, as it is in the allopolyploid parents (Ehrendorfer, 1959). The bivalents of the pentaploid hybrid apparently consist of homologous chromosomes, one from each parent. This, in turn, suggests that the parental populations share homologous genomes. Hence the following phylogenetic relationship and genomic formulas are proposed: $A$. millefolium s. str. $(6 x)$ AASSTT $\times$ A. collina $(4 x)$ AASS $\rightarrow$ Hybrid $(5 x)$ AASST.

In addition, the third genome from the hexaploid parent seems to have a preferential affinity to one of the two others since in the pentaploid hybrid almost exclusively a two plus one alignment is observed. Alignment of all five homoeologous axes and formation of quadrivalents and pentavalents via pairing switches are the exception.

This interpretation, based exclusively on the meiotic pairing behaviour of our $5 x$-hybrid plant, clearly supports theories on the origin of $A$. collina (4x) and $A$. millefolium s. str. (6x) derived from other evidence (Ehrendorfer, 1959; fig. 9 in Greilhuber and Ehrendorfer, 1988): Achillea collina has 
been successfully synthesized from two closely related diploid species, i.e., the pannonic $A$. aspleniifolia (AA) from humid sites and the pontic A. setacea (SS) from steppe communities. A. collina (AASS) is a taxon wide-spread in continental C. and E. Europe, $A$. millefolium s. str. is common in more suboceanic and mountainous sites. from N. and C. Europe to Siberia. That A. collina $(4 x)$ was involved in the origin of $A$. millefolium s. str. $(6 x)$ is suggested by the close approach in morphology and eco-geography of the former towards the latter. The other possible parental taxon which has contributed to the hexaploid is diploid $A$. asiatica (TT) from the mountains of C. Siberia, related to $A$. setacea. If this postulate is accepted, the genome formula of $\boldsymbol{A}$. millefolium s. str. would be AASSTT. This is in good agreement with the data presented in this paper.

Acknowledgements The authors are grateful to Ms G. Holzinger for skilful technical assistance. The work was supported by Grants P6261 B and P6189B of the Austrian Science Promotion Fund (FWF).

\section{REFERENCES}

ALBINI, S. M. AND JONES, G. H. 1984. Synaptonemal complexassociated centromeres and recombination nodules in plant meiocytes prepared by an improved surface-spreading technique. Expl. Cell Res., 155, 588-592.

ASHLEY, T. AND POCOCK, N. 1981. A proposed model of chromosomal organization in nuclei at fertilization. Genetica, 55, 161-169.

BENNETT, M. D. 1984. Premeiotic events and meiotic chromosome pairing. In Evans, C. W. and Dickinson, H. G. (eds) Symp. Soc. Exp. Biol., 38, Company of Biologists, Cambridge, pp. 87-121.

BROWN, W. V. AND STACK, S. M. 1968. Somatic pairing as a regular preliminary to meiosis. Bull. Torrey Bot. Club, 95, 369-378.

DEL FOSSE, F. E. AND CHURCH, K. 1981. Presynaptic chromosome behaviour in Lilium. I. Centromere orientation and movement during premeiotic interphase in Lilium speciosum cv. Rosemede. Chromosoma, 81, 701-716.

DICKINSON, H. G. 1987. The physiology and biochemistry of meiosis in the anther. Int. Rev. Cytol., 107, 79-109.

EHRENDORFER, F. 1959. Differentiation-hybridization cycles and polyploidy in Achillea. Cold Spring Harbor Symp. quant. Biol., 24, 141-152.

GILliES, C. B. 1984. The synaptonemal complex in higher plants. CRC Crit. Rev. Plant Sci., 2(2), 81-116.

GILLIES, C. B. 1987. The effect of $P h$ gene alleles on synaptonemal complex formation in Triticum aestivum $\times T$. kotschyi hybrids. Theor. appl. Genet., 74, 430-438.

GREILHUBER, J. AND EHRENDORFER, F. 1988. Karyological approaches to plant taxonomy. ISI Atlas of Science: Animal and Plant Sciences, 1, 289-297.
GRELL, R. F. 1969. Meiotic and somatic pairing. In Caspari, E. W. and Raven, A. W. (eds), Genetic Organization, Academic Press, New York, pp. 361-492.

HOLM, P. B. AND WANG, X. 1988. The effect of chromosome $5 \mathrm{~B}$ on synapsis and chiasma formation in wheat, Triticum aestivum cv. Chinese Spring. Carlsberg Res. Commun., 53, 191-208.

JENKINS, G. 1985. Synaptonemal complex formation in hybrids of Lolium temulentum $\times$ Lolium perenne (L.). II. Triploid. Chromosoma, 92, 387-390.

JONES, G. H. 1987. Chiasmata. In Moens P. B. (ed.) Meiosis, Academic Press, Orlando, pp. 213-244

JOSEPH, A. M. AND CHANDLEY, A. C. 1984. The morphological sequence of XY pairing in the Norway rat Rattus norvegicus. Chromosoma, 89, 381-386.

LOIDL, J. 1986. Synaptonemal complex spreading in Allium. II. Tetraploid A. vineale. Can. J. Genet. Cytol., 28, 754-761.

LOIDL, J. 1987. Synaptonemal complex spreading in Allium ursinum: pericentric asynapsis and axial thickenings. $J$. Cell Sci., 87, 439-448.

LOIDL, J. 1988. SC-formation in some Allium species, and a discussion of the significance of SC-associated structures and of the mechanisms for presynaptic alignment. Pl. Syst. Evol., 158, 117-131.

LOIDL, J. 1989. Effects of elevated temperature on meiotic chromosome synapsis in Allium ursinum. Chromosoma, 97, 449-458.

LOIDL, J. AND JONES, G. H. 1986. Synaptonemal complex spreading in Allium. I. Triploid A. sphaerocephalon. Chromosoma, 93, 420-428.

Maguire, M. P. 1977. Homologous chromosome pairing. Phil. Trans. R. Soc. Lond. B., 277, 245-258.

MAGUiRE, M. P. 1988. Interactive meiotic systems. In Gustafson, J. P. and Appels, R. (eds) Chromosome Structure and Function. Impact of New Concepts, Plenum, New York, London, pp. 117-144.

PALMER, R. G. 1971. Cytological studies of ameiotic and normal maize with reference to meiotic pairing. Chromosoma, 35, 233-246.

RASMUSSEN, S. W. 1977. Chromosome pairing in triploid females of Bombyx mori analyzed by three dimensional reconstructions of synaptonemal complexes. Carlsberg Res. Commun., 42, 163-197.

SCHNEIDER, I. 1958. Zytogenetische Untersuchungen an Sippen des Polyploid-Komplexes Achillea millefolium L. s. lat. (Zur Phylogenie der Gattung Achillea I). Österr. Bot. Zeitschr., 105, 111-158.

SPEED, R. M. 1986. Oocyte development in XO foetuses of man and mouse: the possible role of heterologous X-chromosome pairing in germ cell survivial. Chromosoma, 94, 115 124.

SUDMAN, P. D., GREENBAUM, I. F., HALE, D. W. AND SMITH, S. A. 1989. Synaptic adjustment in Peromyscus beatae (Rodentia: Cricetidae) heterozygous for interstitial heterochromatin. Cytogenet. Cell Genet., 50, 1-5.

ŚWITOŃSKI, M., GUSTAVSSON, I., HÖJER, K. AND PLÖEN, L. 1987. Synaptonemal complex analysis of the B-chromosomes in spermatocytes of the silver fox (Vulpes fulvus Desm.). Cytogenet. Cell Genet., 45, 84-92.

SYBENGA, J. 1975. Meiotic Configurations. Springer, Berlin.

therman, E. AND SARTO, G. E. 1977. Premeiotic and early meiotic stages in the pollen mother cells of Eremurus and in human embryonic oocytes. Hum. Genet., 35 , 137-151. 
TRES, L. L. 1977. Extensive pairing of the XY bivalent in mouse spermatocytes as visualized in whole-mount electron microscopy. J. Cell Sci., 25, 1-15.

WANDALL, A. AND SVENDSEN, A. 1985. Transition from somatic to meiotic pairing and progressional changes of the synaptonemal complex in spermatocytes of Aedes aegypti. Chromosoma, 92, 254-264.

WANG, X. 1988. Chromosome pairing analysis in haploid wheat by spreading of meiotic nuclei. Carlsberg Res. Commun., $53,135-166$.
WETTSTEIN, D. VON, RASSMUSSEN, S. W. AND HOLM, P. B. 1984. The synaptonemal complex in genetic segregation. Ann. Rev. Genet., 18, 331-413.

WHITE, J., JENKINS, G. AND PARKER, J. S. 1988. Elimination of multivalents during meiotic prophase in Scilla autumnalis. I. Diploid and triploid. Genome, 30, 930-939. 